\title{
KM3NeT acoustic positioning and detection system
}

\author{
Salvatore Viola ${ }^{1, \star}$ for the KM3NeT Collaboration \\ ${ }^{1}$ INFN - Laboratori Nazionali del Sud
}

\begin{abstract}
In the Mediterranean Sea, the KM3NeT Collaboration is constructing a the deep-sea research infrastructure hosting next generation neutrino telescopes. In the KM3NeT telescopes the Cherenkov radiation induced by the secondary charged particles produced in the interaction of cosmic and atmospheric neutrinos within an effective volume between megaton and several cubic kilometers of water are detected by an array of thousands of photomultipliers. The capability of the telescope to determine the direction of secondary charged particles and to point back to the neutrino source is strongly connected to the accuracy on photomultipliers positions. In KM3NeT, the photomultiplier positions are continuously monitored by an acoustic positioning system, designed by the KM3NeT Collaboration to reach an accuracy of the photomultiplier positions better than $20 \mathrm{~cm}$.
\end{abstract}

\section{Introduction}

The construction of the KM3NeT underwater telescopes in the Mediterranean Sea has started [1]. The Cherenkov light induced by secondary charged particles is detected by an array of thousands of photomultipliers, installed in pressure-resistant glass spheres, referred to as Digital Optical Modules (DOMs), attached to flexible mooring lines anchored on the sea-bottom, called Detection Units (DUs). Each DU hosts 18 DOMs, each containing 31 photomultipliers, several calibration instruments and readout electronics. In order to reconstruct the direction of the charged particles with the precision required by the scientific purposes of the KM3NeT project, the DU base positions must be georeferenced with an uncertainty of about two meters and the relative positions of the DOMs must be continuously monitored with an accuracy better than $20 \mathrm{~cm}$ [2]. These requirements are met through a long baseline (LBL) acoustic system composed of a number of transponders (emitter-receiver couples) installed on fixed positions on the sea-bottom and of an array of time-synchronized piezo-acoustic receivers installed inside the DOMs. Knowing the sound velocity profile along the water column and the time of flight of the acoustic pulses emitted by the LBL transponders to reach each piezo-acoustic receiver, DOM positions are calculated through multi-lateration procedures.

\section{Navigation and absolute positioning system}

To provide relative DOM positioning with an accuracy of about $20 \mathrm{~cm}$, the relative position of the LBL elements must be known with an accuracy of the same order. The absolute position of every

\footnotetext{
^e-mail: sviola@lns.infn.it
} 
LBL elements is provided by the Navigation and Absolute Acoustic Positioning System (NAAPS). NAAPS is operated from ship during sea campaigns for the deployment of underwater structures. It provides geo-referenced positions of DU bases and absolute positions of LBL elements with an accuracy of about 3 metres. Knowing the sound velocity in situ, the LBL position accuracy can be improved by auto-calibration procedures based on range measurements. Starting from the nominal positions provided by the NAAPS, LBL element positions are recalculated through iterative algorithms by considering the fixed relative distances for all hydrophone-emitter couples. LBL emitters are typically coupled to an acoustic receiver installed in the same mechanical structure. The Time of Emission (ToE) is calculated by considering the Time of Arrival (ToA) of the signal to the nearby receiver, mechanically constrained to the emitter. In the first stages of the detector construction only autonomous emitters are used for positioning. In this last case, ToE is recovered from ToA to acoustic receivers mounted on DU bases.

\section{The relative acoustic positioning system}

LBL acoustic signals are acquired by digital acoustic receivers (DARs) distributed within the telescope volume. Two different kinds of DARs are used for positioning purpose. Digital hydrophones are hosted on the base of the DUs. These "external" hydrophones are used to calibrate the LBL by measuring the relative distance among LBL elements. The selected hydrophone is the DG0330 manufactured by Colmar s.r.l. It consists of a spherical piezo-ceramic element read-out by an analogue board, splitting the signal in two lines with different gains ( $+46 \mathrm{~dB}$ and $+26 \mathrm{~dB}$ respectively). The movement of the DUs due to underwater currents is monitored by means of "internal" piezo-electric Digital Acoustic Receivers (DAR) glued inside the glass sphere of each KM3NeT Digital Optical Module (DOM) [3].

The acoustic positioning data analysis is entirely performed on-shore. At the shore station, a computer farm parses acoustic data from the main detector data stream. LBL signal identification is performed in real-time by using software algorithms based on cross-correlation methods. Once a LBL-beacon pulse is identified, the software associates it with the absolute GPS time of the detection (ToA) and with a quality factor.

\section{First results}

Presently, the acoustic positioning system of the KM3NeT detector, deployed at $\sim 3500 \mathrm{~m}$ off the Sicilian coasts, includes 3 autonomous emitters and an array of 17 "internal" DAR installed inside the 17 available operational DOMs. Each emitter generates cycles of 12 acoustic pulses every 10 minutes. Positioning pulses are $5 \mathrm{~ms}$ long sweep signals with a different frequency range for each emitter. ToEs are recovered by the ToAs at internal DAR installed in the lowest DOM used as reference. Assuming the reference DAR at a fixed position, relative positions of all DOMs are independently computed. DOM positions are computed every ten minutes with a statistical error of about $10 \mathrm{~cm}$. Figure 1 reports the movement of the DU during a period of about 6 days (150 hours). The reconstructed DOM positions, show that the DOMs move coherently and exhibit periodical movements in agreement with the expected period of the inertial motions induced by the Coriolis force, that at the latitude of KM3NeT site has a period of about 20 hours. 

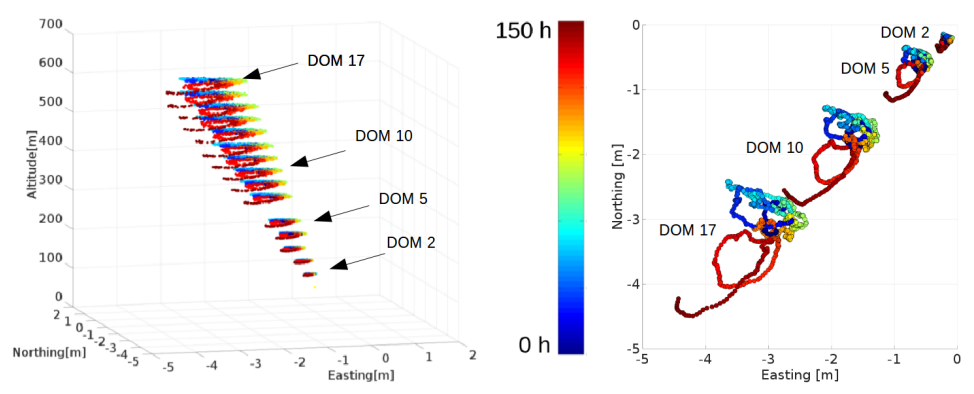

Figure 1. Left: Movements of all available DOMs installed in the operational detection unit at the KM3NeT underwater site of Capo Passero (offthe Sicilian coasts) with respect to the nominal positions. Positions were computed during 150 hours of data acquisition with a time resolution of 10 minutes. The color scale refers to the time from the start of the measurement. Right: Top view of the movements of four reference DOMs. DOM positions have been filtered by using a 2 hours long moving average filter.

\section{Conclusions}

The KM3NeT acoustic positioning system represents the first real-time positioning system at very deep sea in the Mediterranean Sea. It is an evolution of the acoustic positioning system of the ANTARES telescope which is successfully operated for more than 10 years at $2500 \mathrm{~m}$ depth [4].

First results obtained with a minimal system composed of three autonomous emitters and 17 acoustic receivers demonstrated that the position of each optical sensor of the detector can be continuously monitored with a statistical accuracy better than $20 \mathrm{~cm}$, as requested by the detector design. Moreover, thanks to an innovative data acquisition system based on an"all data to shore" philosophy, derived by data acquisition chain implemented in the Submarine Multidisciplinary Observatory (SMO) project [5], data acquired by the acoustic receivers of the KM3NeT positioning system can also be used for the detection and tracking of underwater acoustic sources (natural and anthropogenic) and for the development of innovative techniques for very high energy neutrino detection based on thermal-acoustic model.

\section{References}

[1] KM3NeT Collaboration web site, https://www.km3net.org/

[2] Adrián-Martínez S. et al. , Journal of Physics G: Nuclear and Particle Physics, 43,084001, (2016)

[3] Viola S. et al., Proceedings of Science (ICRC2015),1169, (2015)

[4] Adrián-Martínez S. et al., JINST 7 T08002 (2012)

[5] Viola S. et al., Nuclear Instruments and Methods in Physics Research Section A: Accelerators, Spectrometers, Detectors and Associated Equipment, 725,207-210, (2013) 